\title{
Stability properties and periodic evolutions of bilinear systems in input/output form
}

\author{
Ettore Fornasini* and Maria Elena Valcher \\ Dip. di Elettronica ed Informatica, Univ. di Padova, \\ via Gradenigo 6a, 35131 Padova, Italy \\ e-mail : fornasini@paola.dei.unipd.it
}

\begin{abstract}
Bilinear systems in input/output form are introduced, and represented by means of suitable rational functions in two indeterminates. Necessary and sufficient conditions for BIBO stability are derived, and compared with analogous results on 2D systems stability.

Several features of the output evolutions, corresponding to finite support as well as to periodic input sequences, are discussed, and related to the polar structure of the rational functions that characterize the input-output maps.
\end{abstract}

${ }^{*}$ Author for correspondence. 


\section{Introduction}

In many problems of system analysis and control the physical process is described by means of an input-output (i/o) map, that assigns, to each input, a corresponding output. This situation typically takes place when either the internal complexity of the process is very high or the information available on it is too poor for building up a reliable mathematical model from first principles.

In most cases, a linear time invariant i/o description is adopted. Indeed, identification techniques often produce linear models, and a large number of efficient control algorithms are based on the transfer function approach. On the other hand, when important deviations from linearity and wide excursions of the variables have to be taken into account, nonlinear i/o models could allow for a better fit of the actual behavior. The nonlinear identification problem, however, can be solved satisfactorily only if the class of assumed models is both analytically manageable and large enough to provide a good approximation to the physical process.

An extremely broad catalogue of nonlinear mathematical models is provided by the discrete time version of Volterra series and other related series expansion techniques, that have been investigated by several researchers $[1,2,3,4,5,11]$. In this context, bilinear maps, and the corresponding quadratic maps, represent the simplest instance of nonlinear i/o maps, arising when only the second term of a Volterra series is considered. Actually, several features of the linear case still remain discernable: in particular, when a bilinear map can be realized by finitely many adders, multipliers and delays, a modal analysis of its structure can be performed, that is reminiscent of the classical approach to finite dimensional linear systems. On the other hand, due to the fact that, like $2 \mathrm{D}$ systems in i/o form [6,7], bilinear maps are expressed by power series in two variables, useful insights are also provided by some more unconventional topics of linear multidimensional theory.

The aim of this contribution is to discuss some issues connected with bilinear maps that admit a realization with a finite number of basic elements. We investigate, first, the stability problem and derive necessary and sufficient conditions guaranteeing that bounded inputs always produce bounded outputs. These conditions refer to the polar structure of the rational function that expresses the i/o map, and enlighten some connections among i/o stability of bilinear maps, i/o stability of $2 \mathrm{D}$ transfer functions and the approximately finite memory property. We analyse, next, the free system dynamics, namely the output evolution determined by finite support input sequences. As we shall see, the evolution can be expressed as a linear combination of elementary modes associated with the zeros of suitable polynomials.

Finally, assuming BIBO stability, we consider the asymptotic behavior of the output corresponding to periodic inputs pairs. It turns out that, except for some special cases, the output sequence is eventually periodic, and its permanent component can be determined by resorting to certain diophantine equations. 


\section{The structure of bilinear input-output maps}

In this paper we consider discrete, time invariant, scalar-inputs/scalar-output bilinear system in input/output form. More precisely, we assume that input and output sequences are defined on $\mathbb{N}$ and take values over the real field $\mathbb{R}$, and the output sequence $y(\cdot)$ causally depends on the input pair $\left(u_{1}(\cdot), u_{2}(\cdot)\right)$ according to a map $f(\cdot, \cdot)$ that satisfies the following properties:

$$
\begin{aligned}
f\left(\alpha u_{1}, u_{2}\right)=\alpha f\left(u_{1}, u_{2}\right) & f\left(u_{1}, \beta u_{2}\right)=\beta f\left(u_{1}, u_{2}\right) \\
f\left(u_{1}+v_{1}, u_{2}\right)=f\left(u_{1}, u_{2}\right)+f\left(v_{1}, u_{2}\right) & f\left(u_{1}, u_{2}+v_{2}\right)=f\left(u_{1}, u_{2}\right)+f\left(u_{1}, v_{2}\right),
\end{aligned}
$$

where $\alpha$ and $\beta$ are in $\mathbb{R}$ and $u_{1}, u_{2}, v_{1}$ and $v_{2}$ are arbitrary sequences.

In order to get a concrete representation of $f$, it is convenient to introduce the elementary input pairs $\delta_{i j}(\cdot)$ defined by

$$
\delta_{i j}(t)= \begin{cases}(1,0), & \text { if } t=i \\ (0,1), & \text { if } t=j \\ (0,0), & \text { otherwise }\end{cases}
$$

and to consider the output sequences $s_{k 0}(\cdot)$ and $s_{0 k}(\cdot)$ corresponding to the input pairs $\delta_{k 0}(\cdot)$ and $\delta_{0 k}(\cdot), k=0,1, \ldots$, respectively. Upon arranging the output sequences in the infinite matrix

$$
\mathcal{M}_{f}=\left[\begin{array}{ccccc}
s_{00}(0) & s_{01}(0) & s_{02}(0) & s_{03}(0) & \\
s_{10}(0) & s_{00}(1) & s_{01}(1) & s_{02}(1) & \ddots \\
s_{20}(0) & s_{10}(1) & s_{00}(2) & s_{01}(2) & \ddots \\
s_{30}(0) & s_{20}(1) & s_{10}(2) & s_{00}(3) & \ddots \\
& \ddots & \ddots & \ddots & \ddots
\end{array}\right],
$$

the causality and invariance assumptions imply that the output value at time $t$, corresponding to a generic input pair $\left(u_{1}, u_{2}\right)$, is given by

$$
y(t)=\left[\begin{array}{llll}
u_{2}(t) & u_{2}(t-1) & \cdots & u_{2}(0)
\end{array}\right]\left[\begin{array}{cccc}
s_{00}(0) & s_{01}(0) & \ldots & s_{0 t}(0) \\
s_{10}(0) & s_{00}(1) & \ldots & s_{0, t-1}(1) \\
\ldots & \ldots & \ldots & \ldots \\
s_{t 0}(0) & s_{t-1,0}(1) & \ldots & s_{00}(t)
\end{array}\right]\left[\begin{array}{c}
u_{1}(t) \\
u_{1}(t-1) \\
\vdots \\
u_{1}(0)
\end{array}\right]
$$

Vice versa, every infinite matrix $\mathcal{M}$ induces, via the above construction, a bilinear i/o map.

It is worthwhile to remark that single input/single output quadratic maps can be reduced to the above framework just by associating with a bilinear map $f$ the map $Q_{f}: u \mapsto f(u, u)$. The output value at time $t$ induced by an input sequence $u(\cdot)$ is then given by the quadratic form

$y(t)=\left(Q_{f}(u)\right)(t)=\left[\begin{array}{llll}u(t) & u(t-1) & \cdots & u(0)\end{array}\right]\left[\begin{array}{cccc}s_{00}(0) & s_{01}(0) & \ldots & s_{0 t}(0) \\ s_{10}(0) & s_{00}(1) & \ldots & s_{0, t-1}(1) \\ & \ldots & \ldots & \ldots \\ s_{t 0}(0) & s_{t-1,0}(1) & \ldots & s_{00}(t)\end{array}\right]\left[\begin{array}{c}u(t) \\ u(t-1) \\ \vdots \\ u(0)\end{array}\right]$ 
On the other hand, $Q_{f}$ allows to uniquely retrieve the underlying bilinear map $f$, once the infinite matrix $\mathcal{M}_{f}$ is assumed to be symmetric.

As in the case of linear maps, input and output sequences can be represented by means of formal power series in suitable indeterminates. More precisely, we associate with $\left\{u_{1}(t)\right\}_{t \in \mathbb{N}},\left\{u_{2}(t)\right\}_{t \in \mathbb{N}}$ and $\{y(t)\}_{t \in \mathbb{N}}$ the formal $z^{-1}$-transforms

$$
U_{1}(z):=\sum_{t=0}^{+\infty} u_{1}(t) z^{t} \quad U_{2}(z):=\sum_{t=0}^{+\infty} u_{2}(t) z^{t}, \quad \text { and } \quad Y(z):=\sum_{t=0}^{+\infty} y(t) z^{t}
$$

respectively. Under this assumption, the bilinear i/o map $f: \mathbb{R}^{\mathbb{N}} \times \mathbb{R}^{\mathbb{N}} \rightarrow \mathbb{R}^{\mathbb{N}}$ : $\left(u_{1}, u_{2}\right) \mapsto y$ can be described as follows [8]

$$
Y\left(z_{1} z_{2}\right)=\left(M\left(z_{1}, z_{2}\right) U_{1}\left(z_{1}\right) U_{2}\left(z_{2}\right)\right) \odot \sum_{t=0}^{+\infty}\left(z_{1} z_{2}\right)^{t}
$$

where $M\left(z_{1}, z_{2}\right):=\sum_{i, j=0}^{+\infty} m(i, j) z_{1}^{i} z_{2}^{j}$ is the power series in two indeterminates whose coefficient $m(i, j)$ coincides with $s_{(i-j) \vee 0,(j-i) \vee 0}(i \wedge j)$, the $(i, j)$ th entry of $\mathcal{M}_{f}$, and $\odot$ denotes the Hadamard product of power series.

From a computational point of view, this amounts to saying that the output sequence $\{y(t)\}_{t \in \mathbb{N}}$ coincides with the sequence of "diagonal coefficients" of the Cauchy product

$$
Z\left(z_{1}, z_{2}\right):=M\left(z_{1}, z_{2}\right) U_{1}\left(z_{1}\right) U_{2}\left(z_{2}\right),
$$

which represents the output generated by a $2 \mathrm{D}$ system with transfer function $M\left(z_{1}, z_{2}\right)$, when excited by the input $U_{1}\left(z_{1}\right) U_{2}\left(z_{2}\right)$. In other words, for every $t \in \mathbb{N}, y(t)$ coincides with the diagonal element $z(t, t)$. Notice that, according to our notation, the "diagonal series" $\sum_{i=0}^{+\infty} m(i, i+k)\left(z_{1} z_{2}\right)^{i+k}, k \in \mathbb{N}$, can be identified with the output of (1) corresponding to the canonical inputs $U_{1}(z)=z^{h}$ and $U_{2}(z)=1$, and similarly $\sum_{j=0}^{+\infty} m(j+h, j)\left(z_{1} \cdot z_{2}\right)^{j+h}, h \in \mathbb{N}$, with the output sequence produced by $U_{1}(z)=1$ and $U_{2}(z)=z^{h}$.

A bilinear map (1) can be synthesized by means of a finite number of delay elements, adders and multipliers, namely is "realizable" by interconnecting via multipliers a finite number of time-invariant linear state models, if and only if [8] $M\left(z_{1}, z_{2}\right)$ is a rational power series endowed with the following structure

$$
M\left(z_{1}, z_{2}\right)=\frac{n\left(z_{1}, z_{2}\right)}{h_{0}\left(z_{1} z_{2}\right) h_{1}\left(z_{1}\right) h_{2}\left(z_{2}\right)},
$$

where $h_{0}\left(z_{1} z_{2}\right), h_{1}\left(z_{1}\right)$ and $h_{2}\left(z_{2}\right)$ are $1 \mathrm{D}$ polynomials with nonzero constant terms, while $n\left(z_{1}, z_{2}\right)$ is a $2 \mathrm{D}$ polynomial. Throughout the paper we will assume, without loss of generality, that $h_{0}\left(z_{1} z_{2}\right), h_{1}\left(z_{1}\right)$ and $h_{2}\left(z_{2}\right)$ have unitary constant terms and no common factors with $n\left(z_{1}, z_{2}\right)$.

The structure of (2) is particularly attractive in that it produces a class of bilinear systems that exhibit an autoregressive behavior. Actually, if one rewrites the polynomials 


$$
\begin{array}{lrrr}
h_{0}(z)=1+\alpha_{1} z+\ldots+\alpha_{m_{0}} z^{m_{0}} & h_{1}(z) & =1+\beta_{1} z+\ldots+\beta_{m_{1}} z^{m_{1}} \\
h_{2}(z)=1+\gamma_{1} z+\ldots+\gamma_{m_{2}} z^{m_{2}} & n\left(z_{1}, z_{2}\right) & =\nu_{00}+\nu_{10} z_{1}+\nu_{01} z_{2}+\ldots+\nu_{r s} z_{1}^{r} z_{2}^{s},
\end{array}
$$

the bilinear map can be expressed by means of the following equations

$$
\begin{aligned}
y_{1}(t)+\sum_{i=1}^{m_{1}} \beta_{i} y_{1}(t-i) & =u_{1}(t) \\
y_{2}(t)+\sum_{j=1}^{m_{2}} \gamma_{j} y_{2}(t-j) & =u_{2}(t) \\
y(t)+\sum_{h=1}^{m_{0}} \alpha_{i} y(t-h) & =\sum_{i, j} \nu_{i j} y_{1}(t-i) y_{2}(t-j) .
\end{aligned}
$$

and therefore the bilinear system consists of

- two moving average linear systems, driven by $u_{1}$ and $u_{2}$ and producing two auxiliary outputs $y_{1}$ and $y_{2}$, and

- a linear system, autoregressive in the output $y$ of the bilinear map, and driven by a combination of products of $y_{1}$ and $y_{2}$ taken at different time instants.

\section{BIBO stability}

Consider the bilinear i/o map

$$
\begin{aligned}
f \quad: \quad \mathbb{R}\left[\left[z_{1}\right]\right] \times \mathbb{R}\left[\left[z_{2}\right]\right] & \rightarrow \mathbb{R}\left[\left[z_{1} z_{2}\right]\right] \\
: \quad\left(U_{1}\left(z_{1}\right), U_{2}\left(z_{2}\right)\right) & \mapsto\left(\frac{n\left(z_{1}, z_{2}\right) U_{1}\left(z_{1}\right) U_{2}\left(z_{2}\right)}{h_{0}\left(z_{1} z_{2}\right) h_{1}\left(z_{1}\right) h_{2}\left(z_{2}\right)}\right) \odot \sum_{t=0}^{\infty}\left(z_{1} z_{2}\right)^{t} .
\end{aligned}
$$

The map (3) is said to be bounded input-bounded output (BIBO) stable if for every pair of series, $U_{1}\left(z_{1}\right)$ and $U_{2}\left(z_{2}\right)$, with bounded coefficients, $f\left(U_{1}, U_{2}\right)$ has bounded coefficients, too. BIBO stability of (3) is strictly related to the polar structure of $M\left(z_{1}, z_{2}\right)=n\left(z_{1}, z_{2}\right) /\left[h_{0}\left(z_{1} z_{2}\right) h_{1}\left(z_{1}\right) h_{2}\left(z_{2}\right)\right]$, and, furthermore, it turns out that the above map is BIBO stable if and only if the $2 \mathrm{D}$ i/o map associated with $M\left(z_{1}, z_{2}\right)$ is endowed with this property.

Proposition 1 The bilinear map (3) is BIBO stable if and only if $h_{0}(z), h_{1}(z)$ and $h_{2}(z)$ have no zero in the closed unit disk $D_{1}:=\{z \in \mathbb{C}:|z| \leq 1\}$.

Proof If $h_{0}(z), h_{1}(z)$ and $h_{2}(z)$ have no zero in $D_{1}$, the $2 \mathrm{D}$ transfer function $M\left(z_{1}, z_{2}\right)$ has no singularities in the closed unit polydisk $D_{2}:=\left\{\left(z_{1}, z_{2}\right) \in \mathbb{C}^{2}:\left|z_{1}\right| \leq 1,\left|z_{2}\right| \leq 1\right\}$ and hence is BIBO stable [9]. Consequently, every pair of bounded inputs $\left(U_{1}\left(z_{1}\right), U_{2}\left(z_{2}\right)\right)$ produces a $2 \mathrm{D}$ bounded output $M\left(z_{1}, z_{2}\right) U_{1}\left(z_{1}\right) U_{2}\left(z_{2}\right)$, whose diagonal, i.e., the output $y$, is obviously bounded.

Suppose, now, that (3) is BIBO stable. 
- We prove, first, that $h_{2}(z)$ has no zero in $D_{1}$. If not, we would have $\left(1-\alpha z_{2}\right) \mid h_{2}\left(z_{2}\right)$, for some $\alpha \in \mathbb{C},|\alpha| \geq 1$. Consider the following power series

$$
\begin{aligned}
& U_{1}\left(z_{1}\right)=h_{1}\left(z_{1}\right) \bar{U}_{1}\left(z_{1}\right) \\
& U_{2}\left(z_{2}\right)= \begin{cases}\frac{1}{1-\alpha z_{2}} & \text { if }|\alpha|>1, \\
\frac{1}{\left(1-\alpha z_{2}\right)\left(1-\alpha^{*} z_{2}\right)} & \text { if } \alpha \in \mathbb{C} \backslash \mathbb{R} \text { and }|\alpha|=1\end{cases}
\end{aligned}
$$

where $\alpha^{*}$ is the conjugate of $\alpha$ and $\bar{U}_{1}\left(z_{1}\right)$ is a series, with bounded coefficients, to be determined. Both $U_{1}\left(z_{1}\right)$ and $U_{2}\left(z_{2}\right)$ have bounded coefficients and the corresponding output is given by

$$
Y\left(z_{1} z_{2}\right)=\left(\frac{n\left(z_{1}, z_{2}\right)}{h_{0}\left(z_{1} z_{2}\right)} \bar{U}_{1}\left(z_{1}\right) \frac{U_{2}\left(z_{2}\right)}{h_{2}\left(z_{2}\right)}\right) \odot \sum_{t=0}^{\infty}\left(z_{1} z_{2}\right)^{t} .
$$

If we express $n\left(z_{1}, z_{2}\right)$ as

$$
n\left(z_{1}, z_{2}\right)=z_{1}^{T-1} \tilde{n}_{T-1}\left(z_{2}\right)+z_{1}^{T-2} \tilde{n}_{T-2}\left(z_{2}\right)+\ldots+\tilde{n}_{0}\left(z_{2}\right),
$$

with $T \in \mathbb{N}, \tilde{n}_{i}\left(z_{2}\right) \in \mathbb{R}\left[z_{2}\right], i=0,1, \ldots, T-1$, by the coprimality assumption on the pair $\left(n\left(z_{1}, z_{2}\right), h_{2}\left(z_{2}\right)\right)$, it follows that there exists $j$ such that $\left(1-\alpha z_{2}\right)$ does not divide $\tilde{n}_{j}\left(z_{2}\right)$. Consequently, the coefficients of the power series expansion of

$$
W\left(z_{2}\right):=\frac{\tilde{n}_{j}\left(z_{2}\right) U_{2}\left(z_{2}\right)}{h_{2}\left(z_{2}\right)}
$$

constitute an unbounded sequence, and one at least of the subsequences

$$
\{w(k T)\}_{k},\{w(k T+1)\}_{k}, \ldots,\{w(k T+T-1)\}_{k}
$$

diverges. So, there exists $P \in \mathbb{N}$ such that

$$
\left(\frac{z_{1}^{P}}{1-z_{1}^{T}} \frac{n\left(z_{1}, z_{2}\right) U_{2}\left(z_{2}\right)}{h_{2}\left(z_{2}\right)}\right) \odot \sum_{t}\left(z_{1} z_{2}\right)^{t}
$$

corresponds to an unbounded sequence. It is now clear that, if we assume in (4)

$$
\bar{U}_{1}\left(z_{1}\right):=\frac{z_{1}^{P}}{1-z_{1}^{T}}
$$

the output series in (5) represents an unbounded output.

- The proof that $h_{1}(z)$ has no zero in $D_{1}$ follows the same lines.

- To show that $h_{0}(z)$ has no zero in $D_{1}$, suppose, by contradiction, that there exists $\alpha \in \mathbb{C},|\alpha| \geq 1$, such that $(1-\alpha z) \mid h_{0}(z)$ and express $n\left(z_{1}, z_{2}\right)$ as follows

$$
n\left(z_{1}, z_{2}\right)=z_{1}^{N} n_{N}\left(z_{1} z_{2}\right)+z_{1}^{N-1} n_{N-1}\left(z_{1} z_{2}\right)+\ldots+n_{0}\left(z_{1} z_{2}\right)+\ldots+z_{2}^{M} n_{-M}\left(z_{1} z_{2}\right),
$$


with $n_{i}\left(z_{1} z_{2}\right) \in \mathbb{R}\left[z_{1} z_{2}\right]$ and $N, M \in \mathbb{N}$. As $n\left(z_{1}, z_{2}\right)$ and $h_{0}\left(z_{1} z_{2}\right)$ have no common factor, there exists $i \in\{-M,-M+1, \ldots, N\}$ such that $n_{i}\left(z_{1} z_{2}\right)$ is not a multiple of $1-\alpha z_{1} z_{2}$.

If $|\alpha|>1$ or $|\alpha|=1$ and its multiplicity is greater than 1 , the power series associated with $n_{i}\left(z_{1} z_{2}\right) / h_{0}\left(z_{1} z_{2}\right)$ corresponds to an unbounded sequence, and the output sequence corresponding to the bounded inputs

$\left\{\begin{array}{l}U_{1}\left(z_{1}\right)=h_{1}\left(z_{1}\right) \\ U_{2}\left(z_{2}\right)=h_{2}\left(z_{2}\right) z_{2}^{i}\end{array} \quad\right.$ for $i \geq 0, \quad$ or $\left\{\begin{array}{l}U_{1}\left(z_{1}\right)=h_{1}\left(z_{1}\right) z_{1}^{-i} \\ U_{2}\left(z_{2}\right)=h_{2}\left(z_{2}\right)\end{array} \quad\right.$ for $i<0$

is unbounded.

Assume, now, that the only zeros of $h_{0}(z)$ in $D_{1}$ are simple and of unitary modulus, and let $1 / \alpha$ be one of them. By the coprimality of $n\left(z_{1}, z_{2}\right)$ and $h_{0}\left(z_{1} z_{2}\right)$, it follows that there exists $j$ such that $\left(1-\alpha z_{1} z_{2}\right)$ does not divide $n_{j}\left(z_{1} z_{2}\right)$. Consequently, the coefficients of the series

$$
\sum_{t} w(t)\left(z_{1} z_{2}\right)^{t}=\frac{n_{j}\left(z_{1} z_{2}\right)}{h_{0}\left(z_{1} z_{2}\right)}
$$

do not constitute an $\ell_{1}$ sequence.

Set $T:=N+M+1$. If we assume $j \geq 0$, for instance, and consider the pair of (possibly complex) bounded inputs associated with

$$
U_{1}\left(z_{1}\right)=\frac{h_{1}\left(z_{1}\right)}{1-\left(\alpha z_{1}\right)^{T}} \quad U_{2}\left(z_{2}\right)=\frac{h_{2}\left(z_{2}\right) z_{2}^{j}}{1-z_{2}^{T}},
$$

we have that the output sequence corresponding to the series

$$
\begin{aligned}
Y\left(z_{1} z_{2}\right) & =\left(\frac{n\left(z_{1}, z_{2}\right)}{h_{0}\left(z_{1} z_{2}\right) h_{1}\left(z_{1}\right) h_{2}\left(z_{2}\right)} \frac{h_{1}\left(z_{1}\right)}{1-\left(\alpha z_{1}\right)^{T}} \frac{h_{2}\left(z_{2}\right) z_{2}^{j}}{1-z_{2}^{T}}\right) \odot \sum_{t}\left(z_{1} z_{2}\right)^{t} \\
& =\left(\frac{n_{j}\left(z_{1}, z_{2}\right) z_{1}^{j} z_{2}^{j}}{h_{0}\left(z_{1} z_{2}\right)} \frac{1}{1-\left(\alpha z_{1}\right)^{T}} \frac{1}{1-z_{2}^{T}}\right) \odot \sum_{t}\left(z_{1} z_{2}\right)^{t}=\frac{n_{j}\left(z_{1}, z_{2}\right) z_{1}^{j} z_{2}^{j}}{h_{0}\left(z_{1} z_{2}\right)\left[1-\left(\alpha z_{1} z_{2}\right)^{T}\right]}
\end{aligned}
$$

is unbounded. If $\alpha$ belongs to $\mathbb{R}$ we have obtained in this way a pair of real valued bounded inputs producing an unbounded real output. If $\alpha$ is complex, it is sufficient to assume in (8)

$$
U_{1}\left(z_{1}\right)=\frac{h_{1}\left(z_{1}\right)}{\left[1-\left(\alpha z_{1}\right)^{T}\right]\left[1-\left(\alpha^{*} z_{1}\right)^{T}\right]},
$$

and proceed as before.

Given a $2 \mathrm{D}$ transfer function $N\left(z_{1}, z_{2}\right)=n\left(z_{1}, z_{2}\right) / h\left(z_{1}, z_{2}\right)$, with $n\left(z_{1}, z_{2}\right)$ and $h\left(z_{1}, z_{2}\right)$ factor coprime, if $h\left(z_{1}, z_{2}\right)$ is devoid of zeros in $D_{2}$ the function $M\left(z_{1}, z_{2}\right)$ is $2 \mathrm{D}$ BIBO stable. As it has been proved by D.Goodman [9], however, the converse is not true. Indeed, $M\left(z_{1}, z_{2}\right)$ can be BIBO stable even if it has nonessential singularities of the second kind on the distinguished boundary $T_{2}:=\left\{\left(z_{1}, z_{2}\right) \in \mathbb{C}^{2}:\left|z_{1}\right|=1,\left|z_{2}\right|=1\right\}$ of $D_{2}$, namely if $n(\alpha, \beta)=h(\alpha, \beta)=0$ for some $(\alpha, \beta) \in T_{2}$. When restricting ourselves 
to the class of $2 \mathrm{D}$ transfer functions described as in (2), namely to rational functions which are adopted for representing i/o bilinear maps, 2D BIBO stability is equivalent to assuming that $h_{0}(z), h_{1}(z)$ and $h_{2}(z)$ have no zeros in the closed unit disk $D_{1}$. In fact, if $h_{1}(\alpha)=0,|\alpha|=1$, then $(\alpha, \beta)$ is a zero of the denominator of $M\left(z_{1}, z_{2}\right)$ for every $\beta \in \mathbb{C}$ and hence $M\left(z_{1}, z_{2}\right)$ has nonessential singularities in $D_{2} \backslash T_{2}$, which rules out $2 \mathrm{D}$ BIBO stability. The same reasoning obviously applies to $h_{2}(z)$. On the other hand, if $h_{0}(\alpha)=0,|\alpha|=1$, then all pairs $\left(\alpha e^{i \theta}, e^{-i \theta}\right) \in T_{2}, \theta \in \mathbb{R}$, are zeros of $h_{0}\left(z_{1} z_{2}\right)$, viewed as a $2 \mathrm{D}$ polynomial. As $n\left(z_{1}, z_{2}\right)$ and $h_{0}\left(z_{1} z_{2}\right)$ are factor coprime, and hence have a finite number of common zeros, one at least of the pairs $\left(\alpha e^{i \theta}, e^{-i \theta}\right)$ is a pole of $M\left(z_{1}, z_{2}\right)$. Consequently, the rational function (2) is $2 \mathrm{D}$ BIBO stable if and only if it represents a BIBO stable bilinear i/o map.

It is also worth to remark that BIBO stable bilinear maps are endowed with the "approximately finite memory" (AFM) property introduced by Sandberg in his papers on nonlinear systems $[10,11]$. A bilinear i/o map $f$ has the AFM property if, assuming that $u_{1}(\cdot)$ and $u_{2}(\cdot)$ take values in some finite interval $[-\alpha, \alpha]$ of $\mathbb{R}$, for all $\epsilon>0$ there exists a positive integer $T$ such that $\left|f\left(u_{1}, u_{2}\right)(t)-f\left(W_{t, T} u_{1}, W_{t, T} u_{2}\right)(t)\right|<\epsilon$, where

$$
\left(W_{t, T} u_{i}\right)(\tau):= \begin{cases}u_{i}(\tau), & \text { if } \tau \in[t-T, t] \\ 0, & \text { otherwise. }\end{cases}
$$

$i=1,2$. When $f$ is BIBO stable, the series $M\left(z_{1}, z_{2}\right)$ corresponds to a BIBO stable $2 \mathrm{D}$ system [9], which implies $\sum_{i, j=0}^{+\infty}|m(i, j)|<\infty$. Consequently, given $\epsilon^{\prime}>0$, an integer $N\left(\epsilon^{\prime}\right)$ can be found such that $\sum_{i, j \notin\left[0, N\left(\epsilon^{\prime}\right)\right]}|m(i, j)|<\epsilon^{\prime}$. As the output values $y(t)$ and $\tilde{y}(t)$, produced at time $t$ by the pairs $\left(u_{1}, u_{2}\right)$ and $\left(W_{t, T} u_{1}, W_{t, T} u_{2}\right)$, are given by $\sum_{i, j=0}^{t} u_{1}(i) u_{2}(j) m(t-i, t-j)$ and $\sum_{i, j=t-N\left(\epsilon^{\prime}\right)}^{t} u_{1}(i) u_{2}(j) m(t-i, t-j)$ respectively, when $\left|u_{1}(i)\right|$ and $\left|u_{2}(i)\right|$ are both in $[-\alpha, \alpha]$ for all $i \in \mathbb{N}$ one gets

$$
\begin{aligned}
|y(t)-\tilde{y}(t)| & =\left|\sum_{i, j \notin\left[t-N\left(\epsilon^{\prime}\right), t\right]} u_{1}(i) u_{2}(j) m(t-i, t-j)\right| \\
& \leq \alpha^{2} \sum_{i, j \notin\left[0, N\left(\epsilon^{\prime}\right)\right]}|m(i, j)| \leq \alpha^{2} \epsilon^{\prime},
\end{aligned}
$$

which proves the AFM property.

\section{$4 \quad$ Free and periodic dynamics}

Once a bilinear i/o map $f$ is given, one can exploit shift-invariance to extend $f$ to pairs of inputs with left compact supports, or, equivalently, to pairs of Laurent series. If we consider the space $\mathcal{U}_{1} \times \mathcal{U}_{2}$ of all pairs of inputs whose supports are finite subsets of $(-\infty, 0]$, i.e. the set of trajectories whose power series are elements of $\mathbb{R}\left[z_{1}^{-1}\right] \times \mathbb{R}\left[z_{2}^{-1}\right]$, we can introduce the Nerode equivalence [12] as follows. Two elements $\left(u_{1}, u_{2}\right)$ and $\left(v_{1}, v_{2}\right)$ of $\mathcal{U}_{1} \times \mathcal{U}_{2}$ are said to be Nerode equivalent if for every positive integer $N$ and every pair $\left(w_{1}, w_{2}\right)$, whose support is included in $[1, N]$, the output sequences $f\left(u_{1}+w_{1}, u_{2}+w_{2}\right)$ and $f\left(v_{1}+w_{1}, v_{2}+w_{2}\right)$ coincide in $[N+1,+\infty)$. The classes induced by this equivalence relation are naturally viewed as the states of a "canonical 
realization", and we can identify the outputs of (3) corresponding to finite support input sequences as "free evolutions" corresponding to initial states of the canonical realization.

Let $n\left(z_{1}, z_{2}\right) /\left[h_{0}\left(z_{1} z_{2}\right) h_{1}\left(z_{1}\right) h_{2}\left(z_{2}\right)\right]$ be an irreducible representation of $M\left(z_{1}, z_{2}\right)$ and suppose that $h_{0}(z), h_{1}(z)$ and $h_{2}(z)$ factorize over the complex field in the following way

$$
h_{0}(z)=\prod_{i=1}^{r_{0}}\left(1-\gamma_{i} z\right)^{\rho_{i}} \quad h_{1}(z)=\prod_{i=1}^{r_{1}}\left(1-\alpha_{i} z\right)^{\mu_{i}} \quad h_{2}(z)=\prod_{i=1}^{r_{2}}\left(1-\beta_{i} z\right)^{\nu_{i}},
$$

with $\alpha_{i} \neq \alpha_{j}, \beta_{i} \neq \beta_{j}, \gamma_{i} \neq \gamma_{j}$ for $i \neq j$, and $r_{0}, r_{1}, r_{2}, \rho_{i}, \mu_{i}, \nu_{i}$ positive integers. We aim to explicitly relate the elementary modes describing the free evolution of (3) to the parameters $\alpha_{i}, \beta_{i}$ and $\gamma_{i}$ appearing in (9). To reach this goal, we need a couple of technical lemmas.

Lemma 2 For every choice of the nonnegative integers $n, p$ and $q$ the following identity holds

$$
\left(\begin{array}{c}
n+p \\
p
\end{array}\right)\left(\begin{array}{c}
n+q \\
q
\end{array}\right)=\sum_{k=0}^{p \wedge q}(-1)^{k}\left(\begin{array}{c}
q \\
k
\end{array}\right)\left(\begin{array}{c}
p+q-k \\
q
\end{array}\right)\left(\begin{array}{c}
n+p+q-k \\
p+q-k
\end{array}\right) .
$$

Proof Rewrite the Vandermonde convolutional formula [13] in the form

$$
\left(\begin{array}{l}
x \\
y
\end{array}\right)=\sum_{k=0}(-1)^{k}\left(\begin{array}{c}
x+q-k \\
y-k
\end{array}\right)\left(\begin{array}{l}
q \\
k
\end{array}\right)
$$

and note that with $x$ replaced by $n+p$ and $y$ by $p$, (11) becomes

$$
\left(\begin{array}{c}
n+p \\
p
\end{array}\right)=\sum_{k=0}(-1)^{k}\left(\begin{array}{l}
q \\
k
\end{array}\right)\left(\begin{array}{c}
n+p+q-k \\
p-k
\end{array}\right)
$$

Consequently, we have

$$
\left(\begin{array}{c}
n+p \\
p
\end{array}\right)\left(\begin{array}{c}
n+q \\
q
\end{array}\right)=\sum_{k=0}(-1)^{k}\left(\begin{array}{l}
q \\
k
\end{array}\right)\left(\begin{array}{c}
n+p+q-k \\
p-k
\end{array}\right)\left(\begin{array}{c}
n+q \\
q
\end{array}\right) .
$$

It is a matter of straightforward computation to check the identity

$$
\left(\begin{array}{c}
n+p+q-k \\
p-k
\end{array}\right)\left(\begin{array}{c}
n+q \\
q
\end{array}\right)=\left(\begin{array}{c}
p+q-k \\
q
\end{array}\right)\left(\begin{array}{c}
n+p+q-k \\
p+q-k
\end{array}\right),
$$

and the proof of the lemma is complete upon replacing (13) in (12).

As it is well-known, the power series expansions of the rational functions $1 /(1-$ $\delta z)^{k+1}, k \in \mathbb{N}$, are given by

$$
\frac{1}{(1-\delta z)^{k+1}}=\sum_{n=0}^{\infty}\left(\begin{array}{c}
n+k \\
k
\end{array}\right)(\delta z)^{n}
$$


The following lemma shows that the Hadamard product of $\frac{1}{(1-\alpha z)^{p+1}}$ and $\frac{1}{(1-\beta z)^{q+1}}$ is a linear combination of power series expansions with similar structure, involving the powers of $\alpha \beta$.

Lemma 3 For every $\alpha$ and $\beta$ in $\mathbb{C}$ and every pair $(p, q)$ of nonnegative integers, we have

$$
\frac{1}{(1-\alpha z)^{p+1}} \odot \frac{1}{(1-\beta z)^{q+1}}=\sum_{k=0}^{p \wedge q}(-1)^{k}\left(\begin{array}{c}
q \\
k
\end{array}\right)\left(\begin{array}{c}
p+q-k \\
q
\end{array}\right) \frac{1}{(1-\alpha \beta z)^{p+q-k+1}},
$$

or, in more compact form,

$$
\frac{1}{(1-\alpha z)^{p+1}} \odot \frac{1}{(1-\beta z)^{q+1}}=\frac{1}{q !} \frac{d^{q}}{d z^{q}}\left[\frac{z^{q}}{(1-\alpha \beta z)^{p+1}}\right]=\frac{1}{p !} \frac{d^{p}}{d z^{p}}\left[\frac{z^{p}}{(1-\alpha \beta z)^{q+1}}\right] .
$$

Proof By applying the previous lemma and the power series expansion in (14), we get

$$
\begin{aligned}
\frac{1}{(1-\alpha z)^{p+1}} \odot \frac{1}{(1-\beta z)^{q+1}} & =\sum_{n=0}^{\infty}\left(\begin{array}{c}
n+p \\
p
\end{array}\right)\left(\begin{array}{c}
n+q \\
q
\end{array}\right)(\alpha \beta z)^{n} \\
& =\sum_{k=0}^{p \wedge q}(-1)^{k}\left(\begin{array}{l}
q \\
k
\end{array}\right)\left(\begin{array}{c}
p+q-k \\
q
\end{array}\right) \sum_{n=0}^{\infty}\left(\begin{array}{c}
p+q+n-k \\
p+q-k
\end{array}\right)(\alpha \beta z)^{n} \\
& =\sum_{k=0}^{p \wedge q}(-1)^{k}\left(\begin{array}{l}
q \\
k
\end{array}\right)\left(\begin{array}{c}
p+q-k \\
q
\end{array}\right) \frac{1}{(1-\alpha \beta z)^{p+q-k+1}} .
\end{aligned}
$$

On the other hand,

$$
\begin{aligned}
& \sum_{k=0}^{p \wedge q}(-1)^{k}\left(\begin{array}{l}
q \\
k
\end{array}\right)\left(\begin{array}{c}
p+q-k \\
q
\end{array}\right) \frac{1}{(1-\alpha \beta z)^{p+q-k+1}}= \\
= & \frac{1}{q !} \sum_{k=0}^{p \wedge q}(-1)^{k}\left(\begin{array}{l}
q \\
k
\end{array}\right) \frac{(p-k+1)(p-k+2) \ldots(p-k+q)}{(1-\alpha \beta z)^{p+q-k+1}} \\
= & \frac{1}{(\alpha \beta)^{q} q !} \frac{d^{q}}{d z^{q}}\left[\frac{1}{(1-\alpha \beta z)^{p+1}} \sum_{k=0}^{p \wedge q}\left(\begin{array}{l}
q \\
k
\end{array}\right)(\alpha \beta z-1)^{k}\right] .
\end{aligned}
$$

If $q=p \wedge q$, the summation in (17) gives $(\alpha \beta z)^{q}$, thus proving (16); otherwise, when $p=p \wedge q$, then

$$
\frac{1}{(1-\alpha \beta z)^{p+1}} \sum_{k=0}^{p \wedge q}\left(\begin{array}{l}
q \\
k
\end{array}\right)(\alpha \beta z-1)^{k}=\frac{(\alpha \beta z)^{q}}{(1-\alpha \beta z)^{p+1}}+m(z),
$$

where $m(z) \in \mathbb{R}[z]$ is a polynomial of degree smaller than $q$. So, (16) holds also in this case. 
The above lemma has a significant system theoretic interpretation. Indeed, given a linear system whose transfer function has a pole in $1 / \delta$, the coefficients of the power series expansion in (14) can be regarded as describing a mode associated with the pole. So, the identities (15) and (16) clarify that the Hadamard product of the modes corresponding to the poles $1 / \alpha$ and $1 / \beta$, is a combination of modes corresponding to a pole in $1 /(\alpha \beta)$.

This result is useful for analyzing the free evolution of the bilinear model (3). Actually, assume that $U_{1}\left(z_{1}\right)$ and $U_{2}\left(z_{2}\right)$ are polynomial inputs and consider the corresponding output series

$$
Y\left(z_{1}, z_{2}\right)=\frac{1}{h_{0}\left(z_{1} z_{2}\right)}\left(\frac{n\left(z_{1}, z_{2}\right) U_{1}\left(z_{1}\right) U_{2}\left(z_{2}\right)}{h_{1}\left(z_{1}\right) h_{2}\left(z_{2}\right)} \odot \sum_{t}\left(z_{1} z_{2}\right)^{t}\right) .
$$

Set $n\left(z_{1}, z_{2}\right):=\sum_{i, j} n_{i j} z_{1}^{i} z_{2}^{j}$. By resorting to partial fraction expansions and to the factorizations of $h_{0}, h_{1}$ and $h_{2}$ given in (9), we get

$$
\frac{n\left(z_{1}, z_{2}\right) U_{1}\left(z_{1}\right) U_{2}\left(z_{2}\right)}{h_{1}\left(z_{1}\right) h_{2}\left(z_{2}\right)}=\sum_{i, j} n_{i j}\left[p_{1 i}\left(z_{1}\right)+\sum_{r, s} \frac{\rho_{i r s}}{\left(1-\alpha_{r} z_{1}\right)^{s}}\right]\left[p_{2 j}\left(z_{2}\right)+\sum_{v, t} \frac{\tau_{j v t}}{\left(1-\beta_{v} z_{2}\right)^{t}}\right]
$$

with $p_{1 i}\left(z_{1}\right) \in \mathbb{R}\left[z_{1}\right]$ and $p_{2 j}\left(z_{2}\right) \in \mathbb{R}\left[z_{2}\right]$. It is not difficult to check that the Hadamard product of $\sum_{t}\left(z_{1} z_{2}\right)^{t}$ with the above expression gives

$$
\frac{n\left(z_{1}, z_{2}\right) U_{1}\left(z_{1}\right) U_{2}\left(z_{2}\right)}{h_{1}\left(z_{1}\right) h_{2}\left(z_{2}\right)} \odot \sum_{t}\left(z_{1} z_{2}\right)^{t}=p\left(z_{1} z_{2}\right)+\left[\sum_{r, s, v, t} \frac{n_{i j} \rho_{i r s} \tau_{j v t}}{\left(1-\alpha_{r} z_{1}\right)^{s}\left(1-\beta_{v} z_{2}\right)^{t}}\right] \odot \sum_{t}\left(z_{1} z_{2}\right)^{t},
$$

with $p\left(z_{1} z_{2}\right) \in \mathbb{R}\left[z_{1} z_{2}\right]$. As

$$
\left.\frac{1}{\left(1-\alpha_{r} z_{1}\right)^{s}\left(1-\beta_{v} z_{2}\right)^{t}} \odot \sum_{t}\left(z_{1} z_{2}\right)^{t} \equiv \frac{1}{\left(1-\alpha_{r} z\right)^{s}} \odot \frac{1}{\left(1-\beta_{v} z\right)^{t}}\right|_{z=z_{1} z_{2}},
$$

Lemma 3 applies and we get

$$
Y\left(z_{1}, z_{2}\right)=\frac{1}{h_{0}\left(z_{1} z_{2}\right)}\left[p\left(z_{1} z_{2}\right)+\sum_{r, s, v, t} \frac{c_{r s t v k}}{\left(1-\alpha_{r} \beta_{v} z_{1} z_{2}\right)^{s+t-1-k}}\right]
$$

where $c_{r s t v k}:=(-1)^{k}\left(\begin{array}{c}s-1 \\ k\end{array}\right)\left(\begin{array}{c}s+t-2-k \\ s-1\end{array}\right) \sum_{i, j} \rho_{i r s} \tau_{j u t} n_{i j}$. By expressing (18) as sum of partial fractions, we obtain the output sequence $y$ as a linear combination of elementary modes associated with the poles $1 / \gamma_{i}$ and $1 /\left(\alpha_{r} \beta_{v}\right)$.

Interestingly enough, the case possibly occurs that, even though some zeros of $h_{1}(z)$ belong to the interior of $D_{1}$, all products $1 /\left(\alpha_{r} \beta_{v}\right)$ belong to $\mathbb{C} \backslash D_{1}$. Under this assumption, when all zeros of $h_{0}(z)$ are in $\mathbb{C} \backslash D_{1}$, the bilinear system (3) exhibits only convergent modes, although it is not BIBO stable. 
Example 1 Assume in (1)

$$
M\left(z_{1}, z_{2}\right)=\frac{1}{\left(1-1 / 3 z_{1} z_{2}\right)\left(1-2 z_{1}\right)\left(1-1 / 4 z_{2}\right)} .
$$

This i/o bilinear map is not BIBO stable, because $h_{1}\left(z_{1}\right)=1-2 z_{1}$ has a zero inside $D_{1}$. On the other hand, by resorting to the Euclidean algorithm, every pair of finite support inputs can be written as

$$
\left.\left(U_{1}\left(z_{1}\right), U_{2}\left(z_{2}\right)\right)=\left(a\left(z_{1}\right)\left(1-2 z_{1}\right)+c, b\left(z_{2}\right)\left(1-1 / 4 z_{2}\right)+d\right)\right),
$$

where $a\left(z_{1}\right)=\sum_{i} a_{i} z_{1}^{i}, b\left(z_{2}\right)=\sum_{i} b_{i} z_{2}^{i}$, and $a_{i}, b_{i}, c, d \in \mathbb{R}$. So, the corresponding output $Y\left(z_{1} z_{2}\right)$ is given by

$$
\begin{aligned}
Y\left(z_{1}, z_{2}\right) & =\frac{1}{1-1 / 3 z_{1} z_{2}}\left(a\left(z_{1}\right) b\left(z_{2}\right)+\frac{c b\left(z_{2}\right)}{1-2 z_{1}}+\frac{d a\left(z_{1}\right)}{1-1 / 4 z_{2}}+\frac{c d}{\left(1-2 z_{1}\right)\left(1-1 / 4 z_{2}\right)}\right) \odot \sum_{t}\left(z_{1} z_{2}\right)^{t} \\
& =\frac{1}{1-1 / 3 z_{1} z_{2}}\left(\sum_{i} a_{i} b_{i}\left(z_{1} z_{2}\right)^{i}+\sum_{i} c b_{i} 2^{i}\left(z_{1} z_{2}\right)^{i}+\sum_{i} \frac{d a_{i}}{4^{i}}\left(z_{1} z_{2}\right)^{i}+\sum_{i} \frac{c d}{2^{i}}\left(z_{1} z_{2}\right)^{i}\right),
\end{aligned}
$$

and hence is always convergent.

We conclude this section by surveying some aspects of bilinear i/o maps which are connected with their limiting behavior, when a periodic excitation is applied. If we assume BIBO stability, it is easy to realize that (a part from an exceptional set of periodic inputs) any pair of inputs of period $T$ eventually induces a nonzero output with the same period. $u_{2}$, we can always Actually, suppose that $U_{1}\left(z_{1}\right)$ and $U_{2}\left(z_{2}\right)$ are given by

$$
U_{1}\left(z_{1}\right)=\frac{p_{1}\left(z_{1}\right)}{1-z_{1}^{T}}, \quad U_{2}\left(z_{2}\right)=\frac{p_{2}\left(z_{2}\right)}{1-z_{2}^{T}}, \quad \operatorname{deg} p_{1}, \operatorname{deg} p_{2}<T .
$$

If $n\left(z_{1}, z_{2}\right)$ is expressed as in (7), we have

$$
\begin{aligned}
Y\left(z_{1} z_{2}\right) & =\sum_{i=0}^{N} \frac{z_{1}^{i} n_{i}\left(z_{1} z_{2}\right)}{h_{0}\left(z_{1} z_{2}\right)}\left[\frac{p_{1}\left(z_{1}\right)}{h_{1}\left(z_{1}\right)\left(1-z_{1}^{T}\right)} \frac{p_{2}\left(z_{2}\right)}{h_{2}\left(z_{2}\right)\left(1-z_{2}^{T}\right)}\right] \odot \sum_{t}\left(z_{1} z_{2}\right)^{t} \\
& +\sum_{i=1}^{M} \frac{z_{2}^{i} n_{-i}\left(z_{1} z_{2}\right)}{h_{0}\left(z_{1} z_{2}\right)}\left[\frac{p_{1}\left(z_{1}\right)}{h_{1}\left(z_{1}\right)\left(1-z_{1}^{T}\right)} \frac{p_{2}\left(z_{2}\right)}{h_{2}\left(z_{2}\right)\left(1-z_{2}^{T}\right)}\right] \odot \sum_{t}\left(z_{1} z_{2}\right)^{t} \\
& =\sum_{i=0}^{N} \frac{z_{1}^{i} n_{i}\left(z_{1} z_{2}\right)}{h_{0}\left(z_{1} z_{2}\right)}\left[\left(\frac{v_{10}\left(z_{1}\right)}{\left(1-z_{1}^{T}\right)}+\frac{t_{10}\left(z_{1}\right)}{h_{1}\left(z_{1}\right)}\right)\left(\frac{z_{2}^{i} v_{2 i}\left(z_{2}\right)}{\left(1-z_{2}^{T}\right)}+\frac{t_{2 i}\left(z_{2}\right)}{h_{2}\left(z_{2}\right)}\right)\right] \odot \sum_{t}\left(z_{1} z_{2}\right)^{t} \\
& +\sum_{i=1}^{M} \frac{z_{2}^{i} n_{-i}\left(z_{1} z_{2}\right)}{h_{0}\left(z_{1} z_{2}\right)}\left[\left(\frac{z_{1}^{i} v_{1 i}\left(z_{1}\right)}{\left(1-z_{1}^{T}\right)}+\frac{t_{1 i}\left(z_{1}\right)}{h_{1}\left(z_{1}\right)}\right)\left(\frac{v_{20}\left(z_{2}\right)}{\left(1-z_{2}^{T}\right)}+\frac{t_{20}\left(z_{2}\right)}{h_{2}\left(z_{2}\right)}\right)\right] \odot \sum_{t}\left(z_{1} z_{2}\right)^{t},
\end{aligned}
$$

where $v_{1 i}, v_{2 i}, t_{1 i}, t_{2 i}$ are polynomial solutions of the diophantine equations [14]

$$
\begin{array}{rlrl}
\left(1-z_{1}^{T}\right) t_{1 i}\left(z_{1}\right)+z_{1}^{i} h_{1}\left(z_{1}\right) v_{1 i}\left(z_{1}\right) & =p_{1}\left(z_{1}\right), & & i=0,1, \ldots, M, \\
\left(1-z_{2}^{T}\right) t_{2 i}\left(z_{2}\right)+z_{2}^{i} h_{2}\left(z_{2}\right) v_{2 i}\left(z_{2}\right) & =p_{2}\left(z_{2}\right), & i=0,1, \ldots, N .
\end{array}
$$


By the BIBO stability assumption, for large values of the time variable the behavior of the output sequence does not depend on the terms $t_{1 i} / h_{1}$ and $t_{2 i} / h_{2}$ and, consequently, the power series expansion of

$$
\frac{1}{h_{0}\left(z_{1} z_{2}\right)\left(1-\left(z_{1} z_{2}\right)^{T}\right)}\left\{\sum_{i=0}^{N} n_{i}\left(z_{1} z_{2}\right)\left[v_{10}\left(z_{1}\right) v_{2 i}\left(z_{2}\right) \odot \sum_{t}\left(z_{1} z_{2}\right)^{t}\right]+\sum_{i=1}^{M} n_{-i}\left(z_{1} z_{2}\right)\left[v_{1 i}\left(z_{1}\right) v_{20}\left(z_{2}\right) \odot \sum_{t}\left(z_{1} z_{2}\right)^{t}\right]\right\}
$$

asymptotically fits the actual output of the system. Upon setting

$p(z):=\sum_{i=0}^{N} n_{i}\left(z_{1} z_{2}\right)\left[v_{10}\left(z_{1}\right) v_{2 i}\left(z_{2}\right) \odot \sum_{t}\left(z_{1} z_{2}\right)^{t}\right]+\left.\sum_{i=1}^{M} n_{-i}\left(z_{1} z_{2}\right)\left[v_{1 i}\left(z_{1}\right) v_{20}\left(z_{2}\right) \odot \sum_{t}\left(z_{1} z_{2}\right)^{t}\right]\right|_{z=z_{1} z_{2}}$,

the output series can be rewritten as

$$
Y(z)=\frac{p(z)}{h_{0}(z)\left(1-z^{T}\right)}=\frac{t(z)}{h_{0}(z)}+\frac{v(z)}{1-z^{T}},
$$

where $(v(z), t(z))$ is a polynomial solution of the diophantine equation

$$
\left(1-z^{T}\right) t(z)+h_{0}(z) v(z)=p(z)
$$

satisfying $\operatorname{deg} v<T$. Again, the BIBO stability assumption can be used to show that the term $t(z) / h_{0}(z)$ decays asymptotically to zero. Thus the output is eventually periodic, and its permanent evolution is given by the expansion of $v(z) /\left(1-z^{T}\right)$. Note that, as $v(z)$ has degree smaller than $T$, its coefficents give the restriction to a period of the permanent part of the output sequence.

Remark As mentioned at the beginning of the section, the case possibly occurs that a nonzero pair of periodic inputs produces a zero permanent output. This happens when in (19) the polynomial $p(z)$ is a multiple of $1-z^{T}$, and hence, by the BIBO stability assumption, the whole output sequence asymptotically decays to zero.

The following examples enlighten two possible situations when this phaenomenon arises. In the former, the denominators of the periodic inputs simplify with the polynomial $n\left(z_{1}, z_{2}\right)$; in the latter, the supports of the periodic inputs do not intersect.

Example 2 Consider the rational function

$$
M\left(z_{1}, z_{2}\right)=\frac{1-\left(z_{1} z_{2}\right)^{2}}{h_{0}\left(z_{1} z_{2}\right)\left(1-\alpha z_{1}\right)\left(1-\beta z_{2}\right)},
$$

with $\alpha, \beta \in \mathbb{R}$ and all zeros of $h_{0}(z)$ outside $D_{1}$. Corresponding to the pair of periodic inputs

$$
\left(U_{1}\left(z_{1}\right), U_{2}\left(z_{2}\right)\right)=\left(\frac{1-\alpha z_{1}}{1-z_{1}^{2}}, \frac{1-\beta z_{2}}{1-z_{2}^{2}}\right),
$$


we get the output series

$$
\begin{aligned}
Y\left(z_{1}, z_{2}\right) & =\frac{1-\left(z_{1} z_{2}\right)^{2}}{h_{0}\left(z_{1} z_{2}\right)\left(1-z_{1}^{2}\right)\left(1-z_{2}^{2}\right)} \odot \sum_{t=0}^{\infty}\left(z_{1} z_{2}\right)^{t} \\
& =\frac{1-\left(z_{1} z_{2}\right)^{2}}{h_{0}\left(z_{1} z_{2}\right)}\left(\frac{1}{\left(1-z_{1}^{2}\right)\left(1-z_{2}^{2}\right)} \odot \sum_{t=0}^{\infty}\left(z_{1} z_{2}\right)^{t}\right) \\
& =\frac{1-\left(z_{1} z_{2}\right)^{2}}{h_{0}\left(z_{1} z_{2}\right)} \frac{1}{1-\left(z_{1} z_{2}\right)^{2}}=\frac{1}{h_{0}\left(z_{1} z_{2}\right)},
\end{aligned}
$$

thus proving that $y$ asymptotically decays to zero.

Example 3 Consider the rational function

$$
M\left(z_{1}, z_{2}\right)=\frac{1}{h_{0}\left(z_{1} z_{2}\right)},
$$

where $h_{0}(z)$ has all zeros out of $D_{1}$. The pair of periodic inputs

$$
\left(U_{1}\left(z_{1}\right), U_{2}\left(z_{2}\right)\right)=\left(\frac{1+z_{1}^{2}}{1-z_{1}^{3}}, \frac{2 z_{2}}{1-z_{2}^{3}}\right),
$$

produces the output series

$$
Y\left(z_{1}, z_{2}\right)=\frac{1}{h_{0}\left(z_{1} z_{2}\right)}\left(\frac{1+z_{1}^{2}}{1-z_{1}^{3}} \frac{2 z_{2}}{1-z_{2}^{3}} \odot \sum_{t=0}^{\infty}\left(z_{1} z_{2}\right)^{t}\right)=0 .
$$

\section{References}

[1] Fliess M., "Sur les systèmes dynamiques bilinéaires qui sont linéaires", C.R. Sciences, Acad. Sci. Paris, Tome 278, Série A, 1147-49 (1974)

[2] Schetzen M., The Volterra and Wiener theories of nonlinear systems, Wiley, New York, (1980).

[3] De Figuereido R.J., "A generalized Fock space framework for nonlinear system and signal analysis", IEEE Trans.Circ.Sys., CAS-30, 9, 637-47 (1983)

[4] Sandberg I.W., "Structure theorems for nonlinear systems", Multid.Sys.Signal Proc., 2, 267-286 (1991)

[5] Sandberg I.W., "Uniform approximation and the circle criterion", IEEE Trans.Aut.Contr., AC-38, 10, 1450-58 (1993)

[6] Bose N.K., Multidimensional Systems Theory, D.Reidel Publ. Co., Dordrecht (NL), (1985) 
[7] Kaczorek T., "Two-dimensional linear systems", Lecture notes in control and information sciences, Springer-Verlag, Berlin (1978).

[8] Fornasini E. \& Marchesini G., "Algebraic realization theory of bilinear discrete time input-output maps", J.of Franklin Institute Joint Special Issue with Proceedings of the IEEE, 301, 143-160 (1976).

[9] Goodman D., "Some stability properties of two-dimensional linear shift-invariant digital filters", IEEE Trans. Circ. and Sys. CAS-24, 201-208 (1977).

[10] Sandberg I.W., "Approximation theorems for discrete-time systems", IEEE Trans.Circ.Sys., CAS-38, 5, 564-66 (1991)

[11] Park J. \& Sandberg I.W., "Criteria for the approximation of nonlinear systems", IEEE Trans.Circ.Sys., CAS-39, 8, 673-76 (1992)

[12] Nerode A., "Linear automaton transformations", Proc.Amer.Math.Soc., 9, 541-44 (1958)

[13] Riordan J., Combinatorial Identities, J. Wiley \& Sons, NY, (1968).

[14] Kučera V., Analysis and design of discrete linear control systems, Academia, Prague 1991. 